\title{
La fin du Viribus unitis, entre écriture documentaire et fiction (1918-2018)
}

Das Ende der „Viribus Unitis“, zwischen Dokumentation und Fiktion (1918-2018)

The End of the Viribus Unitis, between Documentation and Fiction (1918-1988)

\section{Daniel Baric}

\section{(2) OpenEdition}

\section{Journals}

Édition électronique

URL : http://journals.openedition.org/austriaca/443

DOI : 10.4000/austriaca.443

ISSN : 2729-0603

Éditeur

Presses universitaires de Rouen et du Havre

Édition imprimée

Date de publication : 1 décembre 2018

Pagination : 233-254

ISBN : 979-10-240-1354-1

ISSN : 0396-4590

\section{Référence électronique}

Daniel Baric, « La fin du Viribus unitis, entre écriture documentaire et fiction (1918-2018)», Austriaca [En ligne], 87 | 2018, mis en ligne le 01 mars 2020, consulté le 29 janvier 2021. URL : http:// journals.openedition.org/austriaca/443 ; DOI : https://doi.org/10.4000/austriaca.443 
Daniel BARIC

Sorbonne Université

\section{La fin du Viribus unitis, entre écriture documentaire et fiction (1918-2018)}

Les derniers instants du Viribus unitis, navire amiral de la marine austro-hongroise qui sombra au large de son port d'attache de Pola/Pula en Istrie à l'aube du $1^{\text {er }}$ novembre 1918 , ont suscité depuis un siècle des réactions qui semblent se différencier selon des lignes de démarcation nationales. Dans la pièce 3. November 1918 de Franz Theodor Csokor ${ }^{1}$, un personnage rescapé de la débâcle, le machiniste Pjotr Kacziuk, à propos duquel une didascalie indique qu'il porte à la visière de sa casquette l'inscription Viribus unitis, rend compte à ses compatriotes incrédules de cette issue improbable : le navire sombra, ou plutôt fut d'abord touché sur son flanc par une torpille, puis coula à $\mathrm{pic}^{2}$. Sur une carte postale produite par la marine italienne en revanche, le moment où le navire amiral de la puissance rivale austro-hongroise est englouti dans les flots apparaît à la fois comme un exemple de bravoure et un événement d'une portée exceptionnelle et fondatrice. Une héroïsation des protagonistes y est clairement à l'œuvre. Au sortir de la première guerre mondiale, du point de vue des autorités italiennes, la disparition du Viribus unitis est d'emblée célébrée comme un symbole, une revanche de la défaite traumatisante subie en 1866 par la marine italienne, éperonnée au large de l'île de Lissa/Vis par les forces navales autrichiennes ${ }^{3}$.

Derrière la question de la stratégie militaire poursuivie par le gouvernement italien et celle de la prouesse technique qui fait ressembler l'équi-

1. Franz Theodor Csokor, 3. November 1918 (1936). Les éditions ultérieures indiquent la date avec un chiffre et non l'adjectif ordinal. Pour une étude détaillée de la pièce et sa réception, voir dans ce volume la contribution de Herta Luise Ott.

2. Le dialogue en rend compte de la manière suivante : "RADOsIN: Gesunken? Das Admiralsschiff? / Kacziuk: Versenkt. / Radosin: Sie lügen! / Kacziuk: Wozu das? Sie wünschen Details? In Pola. Am Allerheiligentag. Mit Zeitminen. Vierhundert Mann über Bord - der Kommandant auch darunter! » (3. November 1918, début de l'acte II).

3. Sur la bataille de Lissa et ses répercussions stratégiques et psychologiques pour les deux protagonistes, voir Olivier Chaline, «Les dépouilles de Venise (1797-1866) », dans Pierre Cabanes (dir.), Histoire de l'Adriatique, Paris, Seuil, 2001, p. 434-436. 
pée à une bataille navale, un touché coulé réussi pour les Italiens, se pose aussi celle d'une réalité humaine, la perte de plusieurs centaines de membres d'équipage, à un moment où, formellement du moins, la guerre aurait dû être finie. La carte postale italienne souligne à souhait le drame : l'effet de sidération, le déséquilibre entre les hommes d'équipage et l'imposante masse du navire, qui chavire pour disparaître dans les flots en dix minutes. En cela, la fin du Viribus unitis est une tragédie, mot que l'on retrouve dans les sources contemporaines ${ }^{4}$, y compris parmi les témoins italiens.

Alors qu'une mémoire italienne de l'événement s'est vite imposée et ramifiée, dans l'espace sud-slave s'est progressivement développée une approche narrative et réflexive, en recherche d'un sens à lui donner. La remémoration y prend la forme d'une hérö̈sation centrée sur le capitaine, dont la disparition offre un matériau documentaire susceptible d'être amplifié par la fiction, pour produire plus d'une interprétation symbolique, qui évolue au gré de la formation et de la disparition des États successeurs de l'Autriche-Hongrie.

\section{Une équipée italienne : couler le Viribus unitis}

Le Viribus unitis avait été lancé le 27 juin 1911. L'événement était de portée mondiale, repris par la presse à travers le globe. Il n'avait donc pas échappé à la marine italienne que ce premier dreadnought $t^{5}$, cuirassé des mers de taille exceptionnelle (152 m de long sur $30 \mathrm{~m}$ de large), n'était que l'avant-courrier d'une série. Il s'agissait du premier élément majeur d'un ambitieux programme naval austro-hongrois, dont l'achèvement était prévu en 1924 et qui aurait dû voir la construction de seize navires au total. Ce temps des chantiers lancés dans la construction de géants de mers correspond à celui de grandes ambitions maritimes, à la fois allemandes et autrichiennes. La programmation navale autrichienne se développait en effet parallèlement à l'effort d'équipement

4. On trouve l'expression « unter besonders tragischen Umständen » dans l'article consacré à chaud à la perte du navire : « Untergang des Schlachtschiffes „Viribus Unitis“", Polaer Tagblatt, 2 novembre 1918, p. 1.

5. Le terme anglais dreadnought, soit " l'intrépide " (faisant référence au Dreadnought, premier navire de guerre propulsé par une turbine à vapeur et disposant d'une artillerie lourde mono-calibre lancé en 1906), était largement en usage jusqu'à la fin de la première guerre mondiale, lorsqu'il fut progressivement remplacé par celui de cuirassé. 


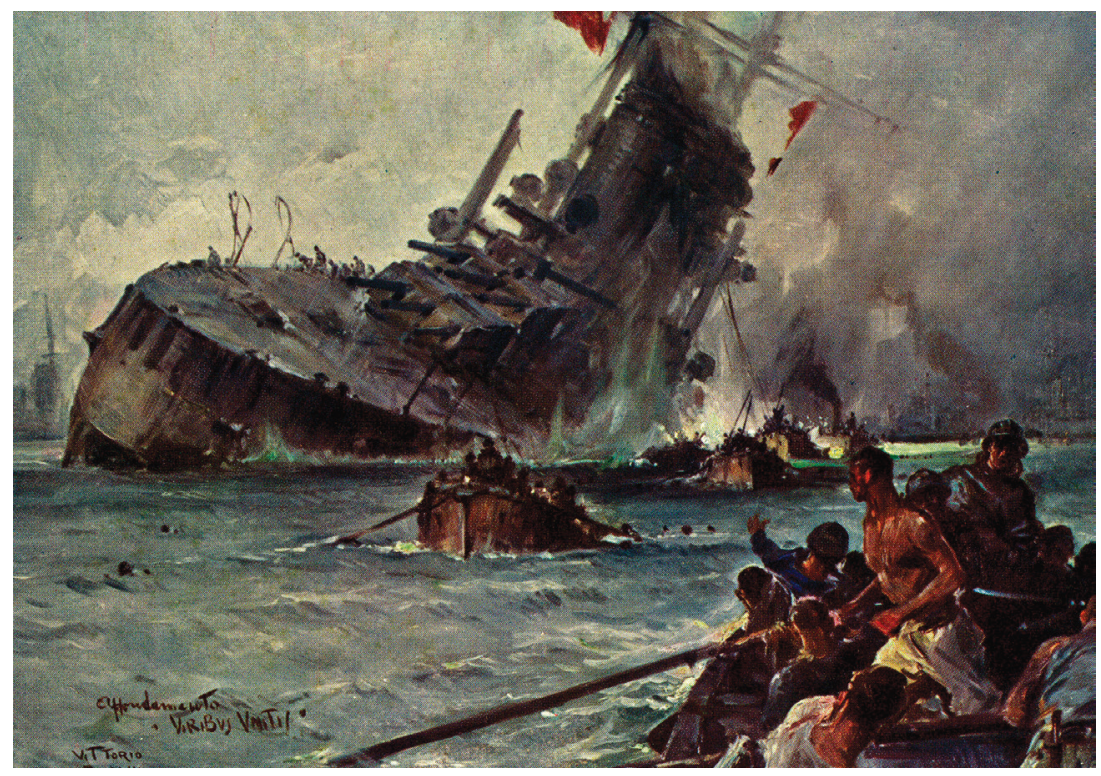

Carte postale italienne. Reproduction du tableau de Vittorio Pisani (1899-1974), Affondamento della "Viribus Unitis ", 1 novembre 1918 [La submersion du "Viribus Unitis»]. Rome, éditions V. E. Boeri, années 1920. Coll. privée.

allemand, la Duplice souhaitant se positionner parmi les premières puissances navales mondiales ${ }^{6}$. À cette course contre la montre que se livraient les grandes puissances européennes pour affirmer leur domination sur les mers se mêlait aussi une question de prestige, non sans un certain emballement. Moins de dix mois après le lancement du Viribus unitis, à la mi-avril 1912, coulait le plus grand paquebot transatlantique du monde, le Titanic, pourtant réputé insubmersible, dans le contexte d'une course qui devait marquer un nouveau record de traversée de l'Atlantique ${ }^{7}$. C'est à Trieste, dans les chantiers navals autrichiens (Sta-

6. Sur cette course aux armements, voir Olivier Chaline, "Amarissimo adriatico", ou la prépondérance maritime autrichienne », dans Pierre Cabanes (dir.), Histoire de l'Adriatique, op. cit., p. 458-465. Sur le programme maritime allemand de Guillaume II en lien avec l'Autriche-Hongrie, voir notamment Christopher Clark, Kaiser Wilhelm II, London, Pearson, 2000, p. 142-138, et Christian Baechler, Guillaume II d'Allemagne, Paris, Fayard, 2003, p. 291-294.

7. Philippe Masson, Le drame du «Titanic », Paris, Tallandier, 1998, p. 13-26. Sur la portée symbolique de ce naufrage lors de sa première traversée, voir David Brunat, Titanic. 
bilimento Tecnico Triestino), qu'avait été construit en deux ans le navire amiral, résultat d'un investissement financier considérable, symboliquement baptisé de la devise de François-Joseph, le souverain incarnant l'Empire : "Toutes les forces unies. " Le prince héritier François-Ferdinand assista au lancement. À la fin juin 1914, trois ans plus tard presque jour pour jour, le cuirassé repassa par Trieste, avec cette fois à son bord les dépouilles mortelles de François-Ferdinand et de son épouse Sophie Chotek, victimes de l'attentat de Sarajevo : son statut prestigieux en faisait le lieu par excellence de l'ostentation du deuil dynastique, catafalque qui longea les côtes de l'Adriatique à l'orée de la première guerre mondiale.

Durant le conflit armé, la guerre navale prit différentes formes : blocus, engagement direct des navires et destruction des flottes ennemies. Elle se poursuivit dans l'espace adriatique jusqu'à la toute fin des hostilités ${ }^{8}$. L'une des modalités de la guerre navale poursuivie par les belligérants consistait à couler les navires ennemis. Pour encadrer (et stimuler) de telles entreprises abondamment relayées par la presse, les autorités italiennes fixent par décret en avril 1918 que les membres d'une opération visant à faire couler un navire ennemi qui serait couronnée de succès toucheraient l'équivalent de $2 \%$ de la valeur nominale du navire 9 . Ainsi le 10 juin 1918 est coulé dans le nord de l'Adriatique, au large de l'île de Premuda, un des bâtiments les plus importants du dispositif mis en place par l'Autriche-Hongrie, le Szent István, un gros porteur construit du côté hongrois de l'Empire, dans les chantiers navals de Fiume/Rijeka ${ }^{10}$. L'événement avait été filmé et les prises de vue montrant les derniers instants de ce cuirassé furent projetées dans l'après-guerre comme un exemple de l'ingéniosité de la marine italienne triomphante. Luigi Rizzo (1887-1951), l'officier à la tête de la flottille qui s'illustra dans le lancement des torpilles, fut fêté comme un héros ${ }^{11}$. L'épave du Szent

Mythe moderne et parabole de notre temps, Paris, Les Belles Lettres, 2013.

8. Sur les opérations de guerre navale, en particulier dans l'espace adriatique, voir Olivier Chaline, "La grande explication, 1912-1918», dans Pierre Cabanes (dir.), Histoire de l'Adriatique, op. cit., p. 486-505.

9. Romain H. Rainero, Raffaele Rossetti. Dall'affondamento della "Viribus Unitis" all'impegno antifascista, Settimo Milanese, Marzorati, 1989, p. 24.

10. Sur la politique hongroise d'armement naval, non sans esprit de concurrence avec les développements en Cisleithanie, voir Mihály Krámli, A császári és királyi haditengerészet és Magyarország. Magyarország szerepe a közös haditengerészet fejlesztésében (La marine de guerre impériale et royale et la Hongrie. Le rôle de la Hongrie dans le développement de la marine de guerre commune), Pécs, Pannónia könyvek, 2004.

11. Voir Fabio Andriola, Luigi Rizzo, Roma, Ufficio Storico della Marina Militare, 2000, p. 101. 
István, échouée par $66 \mathrm{~m}$ de fond, constitue aujourd'hui un terrain de prédilection pour des plongées en archéologie sous-marine ${ }^{12}$. L'officier autrichien Georg von Trapp avait pour sa part coulé en 1915 au large d'Otrante un navire français, le Léon Gambetta, occasionnant plus de 600 victimes. Il rendit compte de cette féroce guerre navale dans ses mémoires, qui n'en furent pas moins traduits en français dans l'entredeux-guerres ${ }^{13}$. Du point de vue militaire, il n'y avait là rien à redire, même du côté français, puisqu'il s'agissait de faits de guerre commis de part et d'autre.

Mais si de tels faits d'armes furent le lot commun de la guerre sous-marine, le sort du Viribus unitis est particulier en ce que le bâtiment fut coulé alors que le conflit était formellement terminé. Le 30 octobre 1918 en effet, l'empereur Charles I ${ }^{\text {er }}$ avait donné ordre aux officiers de la marine austro-hongroise, au contre-amiral Horthy en particulier, qui était en charge de la marine depuis le début du mois de mars, de remettre aux autorités nouvellement constituées dans les territoires sud-slaves de l'Empire, le Comité sud-slave (qui regroupait à Zagreb les représentants des Slovènes, Croates et Serbes de l'Empire), le commandement de la flotte ${ }^{14}$. À ce moment, il s'agissait sans doute pour l'empereur de contribuer à refonder une communauté étatique à partir de différentes entités, sur de nouvelles bases nationales. Quelques semaines auparavant, l'empereur Charles Ir s'était rendu à bord du Viribus unitis. Il y avait eu des échanges avec l'équipage, l'amiral Maksimilijan Njegovan (1858-1930) et le commandant du navire, Janko Vuković von Podkapelski (1871-1918). C'est à ce dernier, dont la loyauté à l'égard de la dynastie était reconnue et qui avait été décoré pour ses mérites dans la guerre sous-marine ${ }^{15}$, que fut remis le lende-

12. Voir Davor Mandić (dir.), Zaštićeni podmorski lokalitet S.M.S. "Szent István" - austrougarski bojni brod klase Tegetthoff (La localisation sous-marine protégée du navire Szent István - navire de guerre austro-hongrois de la classe Tegetthoff), Pula, Povijesni muzej Istre, 2001.

13. Georg von Trapp, Bis zum letzten Flaggenschuss: Erinnerungen eines österreichischen U-Boots-Kommandanten, Salzburg-Leipzig, Pustet, 1935 [Amenez les couleurs! Souvenirs d'un commandant de sous-marin autrichien 1915-1918, Henri Thies (trad.), Paris, Nouvelle Revue critique, 1936].

14. Voir Catherine Horel, L'amiral Horthy, régent de Hongrie, Paris, Perrin, 2014, p. 92 ; Bernard Michel, La Chute de l'Empire austro-hongrois, 1916-1918, Paris, Robert Laffont, p. 254.

15. Vuković avait été décoré en 1915 pour services rendus dans la submersion du contre-torpilleur italien Turbine, qui occasionna la mort de 10 membres d'équipage sur 53 à bord. Voir Bruno Dobrić (dir.), Carska i kraljevska mornarica u Puli/ K.u.K. Marine in Pola, 
main le commandement de l'ensemble de la flotte des mains de Horthy. La réaction locale, dans le port d'attache du navire de Pula, s'inscrit en bandeau de titre sur la première page du Journal croate (Hrvatski List) daté du vendredi $1^{\mathrm{er}}$ novembre 1918, fièrement proclamé " premier jour de la marine yougoslave ». Le 31 octobre y est décrit comme une " journée à marquer d'une pierre blanche dans l'histoire du peuple yougoslave », le journal se félicitant que l'autorité sur l'ensemble des bâtiments « de l'ancien port de guerre autrichien » soit passée dans le calme aux mains des représentants yougoslaves « avec leurs frères tchèques et polonais ${ }^{16}$ ». La décision avait été rendue publique le 30 octobre au soir que le Comité sud-slave de Pula prendrait la direction de la marine au nom des nouvelles autorités de Zagreb. Les articles du journal croate rendent compte en détail des événements de l'après-midi de la journée du 31 octobre, avec en point d'orgue l'acte solennel de remise du commandement des forces navales par Horthy à ses anciens officiers. À 17 heures, " tous les navires hissèrent les pavillons aux couleurs yougoslaves - surtout croates, mais aussi certains aux couleurs slovènes et serbes $^{17}$ ». Le journal ne précise pas que sur les instances de Horthy les couleurs de l'Autriche-Hongrie ne furent amenées qu'une fois l'acte officiel consommé, et non, comme le lui avaient demandé les délégués slaves, avant la rencontre ${ }^{18}$. Le journal, qui paraît tous les jours à 5 heures du matin, est distribué alors que le navire amiral n'a plus qu'une heure et demie d'existence devant lui.

C'est dans une ambiance festive que le Viribus unitis s'était apprêté la veille au soir à passer une première nuit de paix, désormais sous la bannière de l'État sud-slave en voie de formation. À ce moment, deux officiers italiens, Raffaele Rossetti et Raffaele Paolucci, achevaient les préparatifs d'une attaque contre le Viribus unitis prévue de longue date. Le dispositif imaginé ad hoc comprenait la mise au point d'une torpille

catalogue d'exposition, Pula, Sveučilišna knjižnica u Puli/Društvo “Viribus Unitis”, 1999, p. 15.

16. "Prvi dan jugoslavenske mornarice ", " medju svetlije dane jugoslavenskoga naroda ", "pošlo je za rukom da se na miran način preuzme u jugoslavenske ruke, dotično u ruke sa nama sjedinjene braće Čeha i Poljaka sve formacije i sve objekte koli mornarice i brodovlja, toli tvrdjave i ostalih uredjaja bivše austrijske ratne luke Pula " (Hrvatski List, $1^{\text {er }}$ novembre 1918, p. 1). Le terme "Yougoslavie " et l'adjectif " yougoslave », devenus officiels en 1929 pour désigner le Royaume des Serbes, Croates et Slovènes, étaient déjà employés, comme en témoigne notamment la presse de l'époque.

17. «U pet sati posle podne podiglo je celo brodovlje zastave u narodnim jugoslavenskim bojama - ponajviše hrvatske, negde slovenske i srpske » (ibid.)

18. Catherine Horel, L'amiral Horthy, op. cit., p. 94-95. 
équipée d'un moteur qui devait permettre de déjouer la surveillance sur les navires, de s'approcher d'un bâtiment représentatif de la flotte et d'y apposer des explosifs à l'aide d'un type particulier de ventouse ${ }^{19}$. Or en cette soirée et cette nuit du 31 octobre au $1^{\text {er }}$ novembre 1918, pour la première fois depuis le début du conflit, toutes les lumières étaient allumées à bord du Viribus unitis et la surveillance côtière abandonnée. Ce n'est que lorsque les deux Italiens furent à proximité du navire qu'ils furent découverts, soit à 6 heures du matin environ. Faits prisonniers, ils décidèrent de dire la vérité, à savoir qu'ils avaient installé des explosifs qui devaient faire couler le navire. Le commandant Vuković donna immédiatement l'ordre d'évacuer le navire et de conduire sous bonne escorte les deux officiers italiens, qui couraient le risque de voir l'équipage se déchaîner contre eux. L'évacuation commença, mais l'explosion à 6 heures 30 et la rapidité de la montée des eaux dans la quille freina le mouvement. Un peu moins de la moitié de l'équipage put être sauvé. Les chiffres des pertes humaines varient entre 300 et 400 membres d'équipage, qui périrent en quelques minutes, à l'aube du $1^{\mathrm{er}}$ novembre.

Le journal de langue allemande Polaer Tagblatt, qui devait lui-même cesser de paraître peu de temps après ${ }^{20}$, rend compte dans son édition du samedi 2 novembre d'une dépêche radio émise depuis Pula le $1^{\text {er }}$ novembre à 13 heures. Il y est fait appel à l'Italie afin qu'elle cesse ses hostilités à l'égard des Slaves du Sud, puisqu'ils ont pris le pouvoir à la place de l'Autriche-Hongrie. Cette dernière seule ayant été en guerre contre l'Italie, les gouvernements italien et sud-slave devraient se considérer désormais comme alliés. Un appel aux Autrichiens germanophones à effectuer le transport vers l'Autriche " en bon ordre ", en accord avec les autorités yougoslaves et selon les consignes transmises par le Comité des Autrichiens germanophones, se trouve également en première page ${ }^{21}$.

Les récits de la disparition du Viribus unitis dans les flots, à quelques encablures du port, en dehors des opérations entre puissances belligérantes, divergent sur un point essentiel, selon que les Italiens sont réputés

19. Le mot italien utilisé était mignatta, qui peut signifier « anguille » et en l'occurrence «mine ventouse».

20. Le quotidien paraît entre 1905 et 1918, principalement pour les officiers et employés de la Marine (sous le titre Polaer Morgenblatt en 1904-1906). Voir Bruno Dobrić (dir.), K.u.K. Marine, op. cit., p. 27.

21. «An die Deutschösterreicher Polas. Dank dem Entgegenkommen der jugoslawischen Behörden vollzieht sich der Abtransport der Deutschösterreicher in guter Ordnung ", Polaer Tagblatt, 2 novembre 1918. 
avoir ou non eu connaissance que l'Autriche n'était plus, par décision officielle de l'empereur exécutée la veille par Horthy, ni de jure ni de facto la puissance tutélaire du Viribus unitis. La différence est essentielle en ce qu'elle discrimine une action de guerre d'un acte gratuit. Il est remarquable que les deux protagonistes italiens de l'affaire aient développé des interprétations opposées de leur action conçue et effectuée en commun.

\section{Raffaele Rossetti vs. Raffaele Paolucci : communauté d'action et divergences mémorielles}

Au-delà de la réflexion menée en amont et surtout a posteriori sur l'opération contre le Viribus unitis, ce sont les destins sociaux et politiques des deux protagonistes qui divergent. Paolucci (1892-1958), médecin de profession rattaché au corps sanitaire de la marine, rend compte peu après l'événement, dans une revue médicale, de son expérience $^{22}$. Il relate les prémices d'une entreprise qui commence à être ourdie dans les journées de l'offensive austro-hongroise victorieuse de Caporetto de l'automne 1917, alors qu'il se trouve à l'ancre à Malamocco, l'une des entrées de la lagune de Venise.

L'espoir naît en lui de pouvoir venger l'humiliation subie à Caporetto, qui "pesait encore comme une cruelle obsession » : « Et pour la laver, il fallait démontrer que la valeur italienne était étrangère à cette mystérieuse tragédie $e^{23}$. " La préhistoire de l'attaque est mise en relation avec cet épisode douloureux, qui permet d'évoquer des radiotélégrammes envoyés alors depuis le Viribus unitis, "des moqueries et des railleries » vilipendant « ce fou de D'Annunzio ${ }^{24}$ » et qui annon-

22. Raffaele Paolucci, "Relazione sulla spedizione a Pola contro la "Viribus Unitis" ", Annali di medicina navale e coloniale di Roma, $24^{e}$ année, vol. 2, fasc. 5-6, 1918. L'article fut par la suite édité dans L'Esplorazione commerciale, organe mensuel de la société italienne d'explorations géographiques et commerciales, dont le siège est à Milan, sous le titre : "Spedizione per l'affondamento della dreadnought "Viribus Unitis", Pola 31.x.1918-1. XI. $1918 », 36^{e}$ année, fasc. 1-2, 1919, p. 82-95. Les citations sont reprises de cette édition, qui comporte un plan précis du parcours dans la rade de Pola avec la position respective des navires austro-hongrois et des deux Italiens.

23. "L'onta di Caporetto pesava ancora su noi come una ossessione crudele. E per lavarla bisognava dimostrare che il valore italiano era estraneo a quella misteriosa tragedia » (ibid., p. 84).

24. "parole di dileggio e di scherno », " avete finalmente compreso dove vi hanno portato quel pazzo di D’Annunzio?» (ibid., p. 82). 
çaient une avancée austro-hongroise imminente sur Venise, puis Rome. Paolucci déclare avoir pris à ce moment la ferme décision de troquer sa vocation première : «Je fermai les livres de ma bien-aimée chirurgie pour ouvrir ceux sur les torpilles et les missiles, je me rendis maître de tous les mécanismes de ces engins de guerre ${ }^{25}$. " Le modèle qui s'impose alors à lui est celui de Luigi Rizzo, qui " avait donné à la Patrie la victoire de Premuda en coulant le Santo Stefano (Szent István) ${ }^{26} »$. Le premier objectif consiste à couler le navire Radetzky, ancré à l'entrée de la rade de Pula. Il calcule qu'il lui faudrait pour ce faire parcourir une distance de $12 \mathrm{~km}$ à la nage, ramenée bientôt à $8 \mathrm{~km}$, ce qui implique tout de même un entraînement sportif intensif. Il rencontre en juillet le major du génie naval Raffaele Rossetti (1881-1951) avec lequel s'élabore le projet dans les détails opérationnels. Rossetti, d'une " humilité toute franciscaine ", est d'une complexion fort différente de la sienne, il en est bien conscient : " nous étions et sommes deux tempéraments absolument opposés : lui lent, grave, réflexif, taciturne; moi je vais de l'avant, suis rapide en toute action, rieur et $\mathrm{gai}^{27}$. »

Lors de l'opération, ils arrivent en vue de la marine austro-hongroise trois heures avant l'aube, et décident à ce moment de s'attaquer au Viribus unitis qu'ils voient illuminé, plutôt qu'au Radetzky plongé dans l'obscurité. Sans se poser plus de questions avant de passer à l'action, Paolucci affirme que ce n'est qu'une fois à bord qu'ils s'étonnèrent de découvrir des bérets avec l'inscription Jugoslavia et comprirent alors que «[...] la flotte a été "cédée" à la Yougoslavie» :

[...] nous sommes surpris et indécis sur le comportement à adopter : [...] nous vient à l'esprit la possibilité de sauver un grand nombre de vies humaines en reconnaissant que le bâtiment doit périr ; peut-être pourrons-nous nous sauver aussi en avouant la vérité ${ }^{28}$.

25. " chiusi i libri della mia amatissima chirurgia ed aperti quelli delle torpedini e dei siluri, mi resi padrone di tutti i meccanismi di tali ordigni di guerra ( $($ ibid., p. 84).

26. « come preludio alla vittoria del Piave, Luigi Rizzo e Giuseppe Aonzo avevano dato alla Patria la vittoria di Premuda, affondando la "Santo Stefano" » (ibid., p. 85).

27. " francescana umiltà ", " eravamo e siamo due temperamenti assolutamente opposti: egli lento, grave, riflessivo, taciturno ; io sollecito, frettoloso in ogni azione, ilare, giocondo » (ibid.).

28. «la flotta è stata "ceduta" a la Jugoslavia! », « noi siamo sorpresi ed indecisi circa il modo di comportarci $[\ldots]$ appare a nostra mente la possibilità di risparmiare un gran numero di vite umane, confessando che la nave deve perire; forse, confessando la verità, potremo salvarci anche noi » (ibid., p. 93). 
Paolucci saisit dans l'événement quelque chose d'une scène tragique et ne manque pas de mentionner qu'il doit la vie sauve avec son complice au nouveau commandant de la flotte Vuković. Ce dernier décide de les mettre à l'abri de l'équipage, devenu hostile lorsqu'il apprend que le navire vient d'être miné. Sur le refus des deux Italiens de trahir le positionnement exact des explosifs, Vuković commande en allemand à l'équipage de quitter le navire, non sans avoir fait escorter les intrus en canot vers la terre ferme. C'est depuis ce canot de sauvetage que Paolucci observe comment en quelques minutes le navire, après avoir donné de la gîte, s'enfonce inexorablement dans les flots :

Nous nous tournons pour observer la scène tragique. L'aube est déjà là, et dans cette blancheur livide coule la grande masse du Viribus unitis. Le mot unitis est déjà dans l'eau, alors que Viribus émerge encore des flots. Ironie de ce Viribus, qui n'est que faiblesse si décadente sur le point de disparaître ${ }^{29}$.

Le récit mêle la description de la fin du capitaine à celle de l'engloutissement du navire. Paolucci le décrit, dernier à quitter le navire, essayant de rejoindre la côte :

[...] se présente à l'air libre, immense et verdâtre, la quille [du Viribus unitis] qui coule peu à peu. Et sur cette quille je vois un homme qui grimpe, qui rejoint le sommet, qui se tient droit. Je reconnais le commandant Vuković qui mourra peu après, frappé par une poutre alors qu'il cherche à se sauver à la nage, s'étant échappé des remous ${ }^{30}$.

L'engagement militaire n'élimine pas pour autant la formation médicale. Le récit du torpillage est entrecoupé de notations qui trahissent un intérêt marqué pour la physiologie. Il publie du reste ultérieurement plusieurs volumes d'essais médicaux dans ce domaine. Sa description d'une tragédie militaire et humaine est aussi l'occasion de s'observer soi-même comme un cas médical. Son rapport est avant

29. «Ci voltiamo a vedere la tragica scena. L'alba già piena, ed in questo chiarore livido la grande masa della "Viribus Unitis" si sbanda. La scritta "Unitis" è già in acqua, e "Viribus" è ancora emersa. Ironia di quel "Viribus" che è una tanto decadente e rovinante debolezza " (ibid., p. 94).

30. «Riconosco il comandante Vukovic, che morirà poco dopo, colpito da una trave sulla testa, mentre, sottrattosi al gorgo, cerca di salvarsi al nuoto » (ibid., p 94). 
tout professionnel et il rend compte des effets sur son propre corps de cette expédition qu'il observe au ras des flots, à commencer par son entraînement :

Toutes les nuits [...] je me suis exercé dans la lagune. J'eus alors la possibilité de faire une étude sur moi-même de résistance organique à la fatigue consécutive à la natation en eau froide. [...] Le phénomène étrange et pour le moins intéressant était le suivant : je "sentais » mon foie; je le sentais dans sa forme, dans ses dimensions, dans sa position, ses faisceaux ${ }^{31}$.

La douleur diffuse au niveau du foie, les palpitations cardiaques, l'anémie, puis l'administration d'un cordial par des officiers une fois qu'il eut accosté sont relatées avec un soin tout scientifique. La perception d'une "vision tragique " par les deux acolytes est mise en relation avec des « cœurs frappés " par ce spectacle, «affaiblis par une longue souffrance ", qui admirent " combien est humainement beau dans son angoisse » le cri d'un marin qui voit périr son navire bien$a_{i m e ́}{ }^{32}$. Une certaine froideur se dégage de cette évocation, qui du reste est mise à son crédit dans la reconnaissance officielle que lui vaut la mise hors service du navire amiral austro-hongrois : la justification de l'attribution de la médaille d'or du mérite militaire, la distinction la plus honorifique pour hauts faits de guerre, mentionne précisément ce sang-froid dont il semble ne s'être jamais départi tout au long de l'opération et dans les jours qui suivirent, lorsqu'il fut retenu par les autorités sud-slaves avant d'être libéré par l'armée italienne.

Paolucci poursuivit un cursus honorum, à la fois dans le Royaume d'Italie de l'entre-deux-guerres et dans la République italienne. Médecin militaire, il avait débuté une carrière dans l'armée à 26 ans. Promu capitaine par le roi, il est propulsé au sommet de l'État : député durant le fascisme, chef de clinique à Bologne, il est engagé dans les opérations coloniales en Éthiopie. Déchu de ses droits civiques à la fin de la seconde guerre mondiale, bientôt réhabilité, il fut de nouveau élu

31. «Ogni notte [...] esercitai nella laguna. Ebbi agio allora di fare un auto-studio interessante sulla resistenza organica alla fatica del nuoto in acqua fredda [...] Ma fenomeno strano e per lo meno interessante, era questo: io "sentivo" il fegato; lo sentivo nella sua forma, nelle sue dimensioni, nella sua ubicazione, nelle faccie » (ibid., p. 85).

32. «E per i nostri cuori percossi dalla visione tragica, indeboliti dal lungo soffrire, quel grido di disperato amore del marinaio che vede la sua nave perire come sembra umanamente bello nella sua angoscia. La "Viribus Unitis" si sbanda ancora di più, ed appena l'acqua arriva a livello della coperta si capovolge di netto » (ibid., p. 94). 
député, cette fois de la République italienne, en tant que chef du parti monarchiste au Parlement. Paolucci entretint sa vie durant un lien particulier avec ce fait d'armes de sa jeunesse. Ainsi, lorsqu'il eut l'occasion à la fin de sa vie en mai 1951 d'opérer Luigi Rizzo, le héros de ses jeunes années, il souligna qu'il ne put s'empêcher d'admirer le cœur de celui qui sut, impavide, affronter les éléments et les belligérants :

[...] alors que j'opérais à cœur ouvert et que je vis ce cœur qui n'avait pas tremblé en pénétrant dans cette nuit lointaine le port de Trieste pour y couler le Vienne, ce cœur qui n'avait pas tremblé en allant à l'attaque en pleine mer, avec un minuscule mais rapide esquif contre une flotte entière, pour y couler le Saint-Étienne, je me rendis compte que mes yeux se voilaient et je dus m'interrompre un instant ${ }^{33}$.

L'opération chirurgicale qu'il entreprit ne put cependant sauver son camarade.

Raffaele Rossetti mène à bien des égards une carrière inverse. Dès les lendemains de l'équipée, il dut affronter ses supérieurs hiérarchiques, en particulier Costanzo Ciano (1876-1939), le père du futur gendre et ministre des Affaires étrangères de Mussolini, Galeazzo Ciano (19031944). Ce haut gradé chercha en effet à établir sa propre paternité dans le projet. Ce n'est qu'au terme d'une enquête officielle que le mérite (et la récompense) en fut rendu à Rossetti et Paolucci. Rossetti ne cessa dès lors, après un bref soutien aux Arditi partisans de D'Annunzio lors de la prise de Fiume 1919, de s'éloigner de la mouvance fascisante. En 1924, après l'assassinat du député socialiste Giacomo Matteotti, alors que le pouvoir passe aux mains de Mussolini, il publie sa version de la fin du Viribus unitis. Son récit s'inscrit mal dans le projet de l'Italie fasciste : il accuse en effet la marine italienne, non seulement de s'être abstenue de l'aider dans les préparatifs, mais même d'avoir saboté son projet, de l'avoir ralenti par incompréhension et lenteurs organisationnelles, si bien qu'il fut mis en route trop tard. Ce retard apparaît rétrospectivement pour Rossetti comme impardonnable et invalide toute posture héroïque.

33. «Mentre operavo a torace aperto e vidi quel cuore che non aveva tremato entrando nella notte lontana nel porto di Trieste ad affondarvi il "Vienna", quel cuore che non aveva tremato andando all' attacco in pieno mare, con un minuscolo guscio guizzante contro una flotta intera, per affondarvi la "San Stefano", mi accorsi che gli occhi mi si velavano e dovetti un momento sostare » (Fabio Andriola, Luigi Rizzo, op. cit., p. 330-331, d'après Ruggero D’Ondes, Luigi Rizzo l'affondatore, Milazzo, SPES, 1965, p. 334). 
Le livre de souvenirs sur cet épisode crucial de sa vie est sauvé des flammes au début de l'année 1925, alors que l'imprimerie où se prépare l'édition subit un incendie dont les causes ne furent jamais élucidées. Une nouvelle édition ne trouva aucun écho. Rossetti y développe une approche éthique de la question du Viribus unitis. Sa description revient sur les derniers instants du navire et le cri de désespoir d'un marin qui vit disparaître avec son navire, des camarades de l'équipage et le capitaine, comme sur un moment essentiel dans une conversion vers ce qui deviendra un antifascisme assumé, mais non violent :

L'embarcation qui nous a recueillis avance vers la terre ferme ; un des naufragés, recueilli sur la même embarcation que nous, hurle sa douleur dans une langue inconnue de moi et appelle son navire, le Viribus. Mon long effort se dissout en un sentiment de vide et de consternation; devant le terrible spectacle, je me dis : «Et voici mon œuvre! » Peut-être se trouve ici l'origine de la longue crise qui subvertit par la suite toutes mes convictions ${ }^{34}$.

Depuis lors, explique-t-il, la question de l'ennemi politique le préoccupe au plus haut point et il lègue une partie de la somme que lui vaut sa médaille d'or à la veuve de Vuković. Il se retire bientôt de l'armée, devient typographe en exil à Paris, puis rejoint Barcelone pendant la guerre d'Espagne, ce qui lui vaut de perdre la médaille d'or, qui lui sera néanmoins de nouveau décernée après la seconde guerre mondiale. Rossetti se présente sur une liste de gauche après la seconde guerre mondiale, mais n'est pas élu. Une fondation qui porte son nom à Zurich, dont le porte-voix est l'historien suisse Adolf Gasser (1903-1985) qui se place dans le sillage de Denis de Rougemont (1906-1985), veut promouvoir l'exercice de la démocratie directe. La réédition de son ouvrage en 1989, augmentée d'une étude sur son parcours politique, parait alors que se profile le passage au Marché unique européen ${ }^{35}$. Une tradition européenne de vie politique non exclusivement parlementaire y apparaît comme mise en cause par un projet européen trop bureaucratique,

34. «L'imbarcazione che ci ha raccolti procede verso terra; uno dei naufraghi, raccolto dalla stessa imbarcazione che ha raccolto noi, urla il suo dolore in una lingua che non conosco e chiama la sua "Viribus". Il mio lungo sforzo si risolve in un senso vuoto e di costernazione; davanti al tremendo spettacolo, vado dicendo tra me: "E questa è opera mia!" Forse qui è l'inizio della lunga crisi che sovvertì dipoi tutte le mie convizioni » (Raffaele Rossetti, Contro la "Viribus Unitis". Le vicende di un'invenzione di guerra, Roma, Libreria politica moderna, 1925, d'après Romain H. Rainero, Raffaele Rossetti, op. cit., p. 222).

35. Voir Romain H. Rainero, Raffaele Rossetti, op. cit. 
dont l'alternative serait une pratique fortement fédéraliste. La figure de Rossetti, proche d'un Filippo Turati (1857-1932) dans son opposition au fascisme et son isolement politique, demeure au fil des décennies profondément marginale, à l'écart du pouvoir et de ses compromissions.

\section{Mémoires locales : l'héroïsme du vaincu, entre littérature et politique}

La veuve du commandant Vuković fit inscrire cette épitaphe sobre et apolitique au cimetière de Pula : " En souvenir de Janko Vuković-Podkapelski, capitaine de navire de la marine impériale et royale, commandant du Viribus unitis, qui mourut le $1^{\text {er }}$ novembre 1918 d'une mort héroïque dans le port de Pola ${ }^{36}$. » L'inscription est révélatrice d'un mouvement qui s'enclencha immédiatement après la disparition du navire, l'héroïsation de son capitaine. Celle-ci s'opéra sous les instances de la littérature. Ante Tresić Pavičić (1867-1949), écrivain de la génération de Gabriele D'Annunzio (1863-1938), poète, dandy et homme politique issu comme lui du monde adriatique, l'un de la ville de Split, l'autre de Pescara, fut envoyé sur le navire au nom du Comité sud-slave de Zagreb pour y installer officiellement le passage du pouvoir aux nouvelles autorités ${ }^{37}$. Ses discours enflammés reflètent la politisation et la poétisation qui suivirent le passage sous pavillon yougoslave de la flotte austro-hongroise.

Une longue période de silence s'ensuivit durant laquelle le souvenir s'estompa, en raison à la fois de l'éloignement idéologique entre le régime communiste et l'Empire habsbourgeois, et de la transformation démographique que subit la région en un demi-siècle, qui perdit l'essentiel de sa population multilingue. Un capitaine de corvette et journaliste spécialisé dans les affaires militaires qui participa aux combats de la seconde guerre mondiale dans les rangs des partisans de Tito,

36. «Zum Andenken / an / Janko Vuković de Podkapelski / K.u.K. Linienschiffskapitän / Kommandant S.M.S. Viribus Unitis / der am 1. Nov. 1918. im Hafen von Pola den / Heldentod fand. / Die Witwe. "

37. Sur son rôle dans le domaine littéraire et politique, voir Fedora Ferluga-Petronio, Cultura classica e italiana nel Dalmata Ante Tresić Pavičić (1867-1949), Trieste, Centro di studi storico-religiosi Friuli Venezia Giulia, 1992 ; Ivo Frangeš (dir.), Književno djelo Ante Tresića Pavičića (L'œuvre littéraire d'Ante Tresić Pavičić), Split, Književni krug, 1995 ; Ivo Petrinović, Politički život i nazori Ante Tresića Pavičića (La vie politique et les opinions d’Ante Tresić Pavičić), Split, Književni krug, 1997. 
Ivo Ferenca, publia pourtant au milieu des années 1960 des nouvelles historiques qui replacent la marine austro-hongroise et ses participants croates dans une épopée maritime et héroïque. Cette narration s'insère elle-même dans une perspective historique plus largement sud-slave ${ }^{38}$ : la fin du Viribus unitis est aussi celle du " premier commandant de la flotte yougoslave ${ }^{39}$ ». Dernier et tardif acte de guerre, ou bien " première opération de "diversion" contre une nouvelle flotte, qui était en train de naître sur la côte orientale de l'Adriatique. Même les historiens n'ont pas réussi à démêler ce qu'il y a d'exact là-dedans ", écrit Ferenca, qui ne tranche pas, dans un contexte de rapports italo-yougoslaves encore tendus et en attente de statut définitif pour l'Istrie évacuée par les Italiens à la fin de la seconde guerre mondiale ${ }^{40}$. L'écrivain Ivan Katušić (19231984) se rappelle pour sa part avoir encore entendu enfant des histoires de marins de l'époque austro-hongroise. Elles forment l'arrière-plan d'un passé de légendes qu'il retravaille dans des textes dès les années 1960, ce en quoi il apparaît comme un précurseur ${ }^{41}$. Deux reportages en particulier font affleurer cette mémoire qui n'est pas encore revendiquée, mais pour le moins évoquée dans sa dimension plurinationale ${ }^{42}$. Ainsi le cimetière «abandonné » de Pula, celui des marins aux

38. Ivo Ferenca, «U okovima arktičkog leda » (Prisonniers des glaces arctiques), p. 79-100 et «Atentat na zasluženo slavlje» (L'attentat contre une célébration méritée), p. 156-168, dans Željko Grbac (dir.), Đavoli jadranskog mora (Les diables de l'Adriatique), Rijeka, Novi List, 1965. Il s'agit du premier volume d'une série qui devait couvrir la geste des " héros de la mer » (Heroji mora) dans un genre mêlant reportage documentaire et traitement narratif. La collection avait pour ambition d'évoquer le passé lointain, mais aussi de vivifier le souvenir de la résistance locale durant la seconde guerre mondiale, « chapitre sanglant d'une épopée adriatique ", une suite d' "exploits dignes d'une légende » (voir la préface, p. 7).

39. Ivo Ferenca, « Atentat na zasluženo slavlje », art. cité, p. 167-168.

40. Ibid., p. 164.

41. L'auteur croate le plus important de la seconde moitié du $\mathrm{xx}^{\mathrm{e}}$ siècle, Miroslav Krleža (1894-1982), avait quant à lui commencé à s'intéresser à la période habsbourgeoise dans une perspective moins systématiquement critique, avec une sensibilité nostalgique, dans les années de préparation de son monumental roman de la maturité, Les Drapeaux (Zastave, 1962-1968) [Die Fahnen, Gero Fischer et Silvija Hinzmann (trad.), Klagenfurt, Wieser, 2016]. Voir Jan Wierzbicki, Miroslav Krleža, Zagreb, Liber, 1980, p. 62-63.

42. "Pokojna mornarica u bečkom vojnom muzeju " (La défunte marine au musée de l'Armée à Vienne), daté de Vienne, à l'été 1960 (p. 36-39), se présente comme une promenade «à travers l'histoire de cette marine enterrée à Pula en 1918 » ( kroz povijest mornarice koja je pokopana u Puli 1918 », p. 37), et « U boga Posejdona kozje uši (Opet pokojna mornarica) » (Les oreilles de chèvre du dieu Poséidon - de nouveau la défunte marine), daté de Pula, été 1965, p. 43-49, dans Ivan Katušić, Treba putovati - ne treba živjeti. Putopisi i feljtoni (Il faut voyager - il ne faut pas vivre. Récits de voyage et feuilletons), Zagreb, Naprijed, 1966. 
patronymes à consonance germanique notamment, " soumis à la pression conjuguée de la chaleur suffocante et des éclats de voix de la plage voisine met à nu toutes les déceptions allemandes, enterrées ici comme le secret des oreilles de chèvre de Trajan - c'est-à-dire : du dieu Poséi$\mathrm{don}^{43}$ ». Le retour sur les lieux marqués par la marine austro-hongroise et sa fin symbolique s'accompagne d'une introspection :

Bien que je sois né cinq ans après la débâcle de la marine austro-hongroise, je l'ai quand même directement rencontrée et elle devenue une histoire vivante à travers les récits de ses marins, qui dans les jours de mon enfance avaient moins de quarante, et même moins de trente ans. La marine a disparu, ne sont restés que les noms de valeureux officiers et de leurs tortionnaires, les légendes, les commandements et les appels à la trompette, chansons et plaisanteries, les maillots de coton et de laine indéchirables, un humour spécifique au goût de sel ${ }^{44}$.

Or cette communauté humaine semble désormais oubliée de tous, y compris des autorités locales, et singulièrement de la mairie de Pula, qui délaisse ce cimetière, ce qui d'ailleurs, reconnaît l'auteur, lui confère un charme subtil. Dans cet endroit « le point le plus dramatique est assurément la plaque en l'honneur de Janko Vuković de Podkapelski, le premier commandant de la flotte yougoslave », qui, relève-t-il, évoque paradoxalement une mort héroïque pour un empire " ancien et puissant ", « comme si l'Autriche-Hongrie ne s'était pas désintégrée avant même que ne soit coulé le Viribus unitis ${ }^{45}$ ».

Les années 1970 marquèrent en Yougoslavie une césure dans la mémoire du passé austro-hongrois et de la marine en particulier. Alors eurent lieu les premiers essais concrets de réappropriation locale de

43. «Ovo groblje, ovo zapušteno groblje s obližnjeg kupališta otkriva sva germanska razočaranja, koja su ovdje zakopana kao tajna o kozjim ušima cara Trajana - to jest : boga Posejdona » (Ivan Katušić, Treba putovati, op. cit., p. 47).

44. «Iako sam se rodio pet godina nakon što je propala austrougarska mornarica, ipak sam je neposredno susreo i doživio u pričama njezinih mornara, koji su u danima mog djetinstva imali manje od četrdeset, manje od trideset godina. Propade mornarica, a ostadoše imena čestitih oficira i mučitelja, legende, komande i trubni znakovi, pjesme i dosjetke, nepoderive pamučne i vunene maje, specifični, slani morski humor » (ibid., p. 46).

45. «Svakako, najdramatičnija je tačka spomen-ploča Janku Vukoviću Podkapelskom, prvom komandantu jugoslavenske flote ", " nakon propasti starog i moćnog carstva ", « Kao da se Austro-Ugarska nije raspala prije nego što je potopljen Viribus unitis » (ibid., p. 48-49). Katušić avait par ailleurs noté dans un article (« Dva crno-žuta dosjea » [Deux dossiers noir et jaune], daté de 1959) que « les quatre livres des aventures de Chveik forment le monument funéraire durable de la Monarchie austro-hongroise » (Četiri knjige Švejkovih do življaja su trajni nadgrobni spomenik Austro-Ugarskoj monarhiji). Voir ibid., p. 191. 
ce passé austro-hongrois englouti. Pula, ville devenue italienne par les traités d'après-guerre de Rapallo (1920), vidée de sa population italienne lorsqu'elle devint yougoslave en 1945, ce que reconnut le traité italo-yougoslave d'Osimo en 1975, vit ressurgir ce passé impérial. Il se manifesta en particulier par l'établissement de contacts avec les archives autrichiennes, dans le cadre des discussions sur la succession de l'Autriche-Hongrie et les suites de la seconde guerre mondiale. L'une de ces réalisations consista à y faire revenir en 1975 des archives viennoises (Kriegsarchiv) la Bibliothèque de la marine ${ }^{46}$. Le milieu des années 1980 apparaît comme celui d'une redécouverte simultanée, en différents endroits de l'ancien Empire habsbourgeois, de la thématique «mitteleuropéenne ", dont l'un des vecteurs est l'histoire de la marine. Le roman documentaire Les Effrois de la glace et des ténèbres de Christoph Ransmayr (né en 1954) témoigne de ce nouvel intérêt pour le matériau romanesque que recèle un groupe humain jadis important, disparu depuis plusieurs générations ${ }^{47}$. À partir de documents d'archives, dont certains avaient été publiés, le roman élabore, en y incorporant une part de fiction, avec l'insertion d'un personnage de l'invention de l'auteur, le destin collectif d'un équipage multinational. À la recherche du passage du NordOuest à travers les mers froides, les hommes d'équipage, issus pour une grande part des rivages orientaux de l'Adriatique, firent la découverte de l'archipel François-Joseph en $1873^{48}$. La même démarche caractérise le roman d'Ivan Katušić, Admiralski stijeg (L'étendard du vaisseau amiral ${ }^{49}$. Achevée en 1985 et éditée à titre posthume en 1987, lue et commentée dans les dernières années du régime yougoslave, cette œuvre fut conçue principalement sur la base des témoignages des protagonistes italiens. L'auteur s'était procuré l'un des très rares exemplaires du texte de Rossetti en circulation au début des années 1980. Situé à la frontière entre reportage documentaire et fiction, le récit entremêle lui aussi documents historiques et personnages fictionnel $\mathrm{s}^{50}$. Le roman est construit autour de la journée du $1^{\text {er }}$ novembre 1918, dans une chronologie entrecoupée de

46. Roman Lukin, Mornarička biblioteka / K.u.K. Marine-Bibliothek, Pula, Povijesno društvo Istre, 1986.

47. Christoph Ransmayr, Die Schrecken des Eises und der Finsternis, Wien, Brandstätter, 1984 [Les Effrois de la glace et des ténèbres, François Mathieu (trad.), Paris, Seuil, 1991 ; Strahote leda i tame: roman s jedanaest slika, Mladen Udiljak (trad.), Zagreb, Sipar, 2006].

48. Cet épisode avait été traité par Ivo Ferenca, «U okovima arktičkog leda », art. cité.

49. Ivan Katušić, Admiralski stijeg. Roman, Zagreb, Znanje, « hit - biblioteka moderne literature, 183 », 1987.

50. En début d'ouvrage, l'auteur remercie explicitement sa fille pour l'aide apportée dans la 
réflexions sur des documents qui n'existent plus, et qui n'ont sans doute jamais existé : la déposition de deux protagonistes qui devaient préparer le procès de Paolucci et Rossetti. Ces derniers ne furent pas traduits en justice, puisque Pula fut prise par les troupes italiennes le 5 novembre 1918, qui libérèrent les deux officiers et qu'ils passèrent instantanément du statut de prisonniers à celui de héros.

L'ouvrage parut à Zagreb en 1987 chez l'éditeur Znanje, dans la série « Hit ». Cette collection avait déjà publié ou était sur le point de le faire, nombre de textes qui eurent de fait le statut de best-sellers, dans des genres très variés : La Plaisanterie de Milan Kundera, Trains étroitement surveillés de Bohumil Hrabal, mais aussi quelques volumes de la série des don Camillo de Giovannino Guareschi et La Bicyclette bleue de Régine Deforges $^{51}$. En tout état de cause, il s'agit d'un programme éditorial non conforme à une ligne dictée par des impératifs politiques. L'image de couverture, conçue par le graphiste Alfred Pal, reprend sous un bandeau aux couleurs hongroises ${ }^{52}$ une photographie d'époque couleur sépia qui représente le navire amiral au moment où il sombre, retravaillée comme dessin en 1933 par l'artiste Willi Maier, sur laquelle vient s'ajouter en surimpression la photographie d'une horloge qui se trouvait sur le navire. Ces éléments graphiques convergent dans le sens d'un regard renouvelé sur les événements et invitent à la réévaluation de l'aspect tragique de cet événement, et à travers lui de la fin de l'Empire. Le roman évoque la diversité des expériences nationales et l'intensité de ces quelques jours compris entre le $1^{\text {er }}$ et le 4 novembre 1918, entre l'espoir d'une paix durable entre les deux États, italien et yougoslave, la destruction du navire amiral et l'arrivée imminente des troupes italiennes. Comme chez Ransmayr, le détachement d'une perspective nationale et politique est sensible, au profit du document, réel ou fictif, qui donne accès à une

documentation. Également traductrice, elle a fait connaitre des auteurs qui s'inscrivent dans la redécouverte du passé austro-hongrois dans les années 1980. Voir par exemple sa traduction du roman historique d'Anton Hykisch, Volite kraljicu Mariju Tereziju (Milutje Kral'ovnu), 1984, traduit du slovaque par Anka Katušić-Balen, Zagreb, Mladost, 1989.

51. Giovannino Guareschi, Don Camillo i njegovo stado (éd. originale : 1953 ; éd. croate : 1970) et Don Camillo i mladi (1969-1971) ; Milan Kundera, Šala (1967-1982) ; Bohumil Hrabal, Strogo kontrolirani vlakovi (1965-1983) ; Régine Deforges, Plavi bicikl (19811987).

52. Sur le parcours d'Alfred Pal (1920-2010), né à Vienne, qui passa son enfance à Cracovie et Vukovar, fut interné par les Italiens durant la seconde guerre mondiale (dans le camp de Kraljevica et sur l'île de Rab), puis par les autorités yougoslaves sur Goli Otok, voir ses mémoires posthumes édités par Bogdan Žižić, Gorući grm. Alfred Pal - život i djelo (Le buisson ardent. Alfred Pal, vie et œuvre), Zagreb, Durieux, 2011, p. 147-151. 
immédiateté des émotions. Dans les deux cas, l'invention de documents et l'évocation de personnages réels, inscrit la démarche dans un jeu littéraire (le dispositif repose ici sur un manuscrit censément retrouvé chez l'auteur) qui aboutit à une réévaluation de la portée symbolique de la marine austro-hongroise comme creuset de nationalités réunies autour d'un même projet. Dans la construction documentaire à l'œuvre, viennent s'intercaler dans les interstices entre document et fiction des interprétations nouvelles. Chez Katušić, elle se cristallise autour de la figure de Vuković, attaché à la diversité des langues et des origines tout en observant une fidélité sans faille à son devoir. L'écriture documentaire apparaît ici comme une tentative de dire la réalité de manière différente, par un effacement de la ligne claire entre réalité et imaginaire historique. Si le procédé peut assurément être interprété comme le signe d'une crise de la fiction ${ }^{53}$, il s'agit également de l'expression d'une conscience historique à la recherche de nouveaux paradigmes dans une Yougoslavie qui après la mort de Tito en 1980 entrait dans une longue crise du sens même de son existence. Le roman fut favorablement accueilli à sa sortie à partir de 1988, mais la réception fut vite interrompue par les conflits internes, puis les guerres qui s'installèrent dans l'espace yougoslave. Ce premier moment de réception semble aller dans le sens de la reconnaissance, à travers l'éphémère amiral Vuković, de l'incarnation d'un " yougoslavisme » idéal $^{54}$.

Mais l'hérö̈sation progressive de Vuković ne prend pas fin avec la Yougoslavie multiculturelle. Dans une perspective croate, les œuvres qui lui sont consacrées témoignent d'un intérêt renouvelé pour un personnage auréolé de l'image d'un héros fidèle à son devoir. Stjepan Vukušić, un auteur né en 1931, qui put comme Katušić entendre dans sa jeunesse les récits des témoins de la période austro-hongroise sur le littoral adriatique, rend compte dans un roman publié en 2004 de sa fascination pour celui qui lui apparait comme le " premier amiral croate $e^{55}$ ». Habitant de Pula, hanté par le destin de l'amiral d'un demi-jour qui finit sa vie dans

53. Milka Car, Uvod u dokumentarnu književnost (Introduction à la littérature documentaire), Zagreb, Leykam, 2016, p. 91, 127-136.

54. Le parallélisme entre l'Empire austro-hongrois traversé de contradictions et son souverain vieillissant et la Yougoslavie à bout de souffle sous la férule du maréchal Tito a été relevé par les historiens. Voir notamment Joseph Krulic, Histoire de la Yougoslavie, Bruxelles, Complexe, 1993, p. 166-170.

55. Stjepan Vukušić, Admiral. Roman o Janku Vukoviću pl. Podkapelskom, prvom hrvatskom admiralu (L'amiral. Roman sur Janko Vuković de Podkapelski, premier amiral croate), Zagreb, Pavičić, 2004. 
la rade de sa ville, il met en scène Vuković comme " un paradigme ", " une personnalité qui voit la chute d'un empire et la naissance d'un monde nouveau ", en hommage à la "nécropole de Pula ${ }^{56}$ ", dernière sépulture de marins auxquels rend hommage l'épitaphe du roman ${ }^{57}$. À l'appui d'une démarche narrative, l'ouvrage est accompagné de photographies, d'époque et contemporaines, de cartes postales et de schémas. L'auteur revient sur cette figure, non sans introduire un infléchissement par rapport à son prédécesseur Katušić, avec désormais un regard porté sur les signes de son appartenance à l'histoire croate. S'inspirant ouvertement de ce roman, et s'y référant abondamment, le premier amiral de la marine croate après l'indépendance, Davor Domazet-Lošo (1948), rend hommage en 2015 à celui en qui il voit un modèle : l'incipit est consacré à Vuković, à propos duquel il écrit qu'il fut « un homme d'une noble humanité, qui éleva la fonction d'amiral dans des hauteurs inaccessibles, célestes ${ }^{58} »$ Parallèlement à l'inscription dans un horizon étatique, yougoslave puis croate, une incorporation concomitante dans la mémoire régionale de la figure de l'amiral s'est également produite. En 2003, le groupe de rock alternatif GUW (Gori Ussi Winnetou), qui s'est donné pour but d'activer une mémoire istrienne, avait produit une chanson éponyme dans l'album Kobra je u brajdi (Le cobra est dans la treille), entièrement dédié aux figures essentielles, mais marginales de la mémoire régionale, indépendamment de leur appartenance à un groupe linguistique. Le premier titre est consacré à la figure de Vuković. S'y succèdent l'écrivain slovène Ivan Cankar et l'entrepreneur autrichien Paul Kupelweiser, mais aussi Nazario Sauro (1880-1916), irrédentiste italien qui effectua des missions contre la marine austro-hongroise, qui fut capturé, jugé et exécuté à Pula ${ }^{59}$.

La fin du Viribus unitis dans la mémoire locale s'inscrit dans une trame historique, soit par juxtaposition, soit par une technique du montage ${ }^{60}$.

56. Boris Domagoj Biletić (dir.), Književni portret: Stjepan Vukušić (Portrait littéraire de Stjepan Vukušić), Pula, Nova Istra, 2011, p. 16-17.

57. "À tous les marins croates / qui reposent par le fond des mers » («Svim hrvatskim mornarima / koji počivaju na morskome dnu »).

58. « čovjek plemenite ljudskosti, koji je admiralsku čast uzdigao na nedostižne, nebeske visine » (chap. "Admiralska čast »), dans Davor Domazet-Lošo, Admiralovi zapisi ili O pobjedi i ljubavi (Les notes de l'amiral ou Sur la victoire et l'amour), Zagreb, Udruga Hrvatski identitet i prosperitet / Matica hrvatska, ogranak Sinj, 2015, p. 17.

59. "Janko Vuković de Podkapelski - Viribus Unitis (za šaku apaurina) " (Janko Vuković de Podkapelski - Viribus Unitis - pour une poignée d'aspirines), https://www.youtube. com/watch?v=uo9OutO_Ip0 (consulté le 13 avril 2019).

60. Nikolaus Miller, Prolegomena zu einer Poetik der Dokumentarliteratur, München, Fink, 
L'usage de citations, l'insertion d'images et du son rendent compte d'un processus d'hybridation comme mode de création qui ouvre de nouvelles perspectives d'interprétations des événements historiques. Ces démarches artistiques se sont développées en même temps qu'une attention plus importante fut portée aux restes visibles et au souvenir du navire et de son équipage.

Sur les lieux de l'événement, la carcasse du Viribus unitis a été extraite de la rade de Pola dès l'entre-deux-guerres. Le corps de l'amiral Vuković n'y a pas été retrouvé. Une politique mémorielle concertée de conservation du cimetière de la marine s'est progressivement mise en place à partir des années 1960. Inscrit au registre des monuments historiques mais sans financement, un accord multipartite signé en 1990 entre la municipalité de Pula, la Croix-Rouge autrichienne et des représentants allemands de l'association en charge des sépultures des soldats aboutit à un vaste projet de réhabilitation, achevé en 1997. La coopération autour de traces matérielles, à travers la figure de l'amiral qui laissa la vie sauve aux torpilleurs de son navire, commémore tout l'Empire austro-hongrois en tant qu'expérience humaine. Au-delà d'un horizon politique national, c'est le symbole d'une tradition maritime qui est remémoréb1. Indépendamment des modes d'expression et des langues, s'est esquissée de manière de plus en plus marquée depuis les dernières décennies une convergence des mémoires autour de l'héroïsation, au sens d'une insertion au centre du discours et de la narration, des perdants de l'histoire que furent le Viribus unitis, son commandant et ses matelots, la flotte et l'Empire qu'elle servait. Mais à mesure que s'éloigne dans le temps la réalité historique de l'Empire habsbourgeois, l'interprétation de cette mémoire de la fin conjointe d'un navire, d'une marine et d'un empire, peine à se matérialiser.

En Croatie, la fiction supplée au manque d'explication satisfaisante que peut livrer l'histoire documentée. Alors que s'estompe dans l'Italie républicaine le souvenir de l'épisode du Viribus unitis, des ancres encore visibles, notamment à l'entrée du ministère de la Marine italienne à Rome, témoignent de sa présence encore palpable mais sous une forme atténuée de trophée, comme une réplique de la colonne rostrale

p. $50-84$.

61. L'attitude irréprochable de l'amiral est présentée comme un exemple universel, notamment par Heinrich Bayer von Bayersburg (Österreichs Admirale und bedeutende Persönlichkeiten der k.u.k. Kriegsmarine, 1867-1918, Wien, Bergland, 1962, p. 175). 
de Vienne qui célèbre depuis 1886 au Praterstern la victoire de Lissa de 1866 et son héros Tegetthoff. À Vienne, le souvenir du Viribus unitis est certes maintenu également au musée de l'Armée, où l'on peut en voir une maquette en découpe, un travail entrepris par des marins. Mais une marque d'horlogerie autrichienne a récemment tenté de réactiver cette mémoire, sans doute quelque peu diluée, en se nommant «Viribus Unitis » et avec pour logo les initiales du navire amiral. Les produits proposés, " au design élaboré dans la Forêt viennoise ", sont des montres destinées " aux vrais hommes, légendes en marche », qui reprennent en éditions limitées des modèles en usage dans l'armée austro-hongroise. La commercialisation d'une gamme de montres étanches, particulièrement résistantes à la pression sous-marine, était annoncée pour la fin de l'année $2018^{62}$.

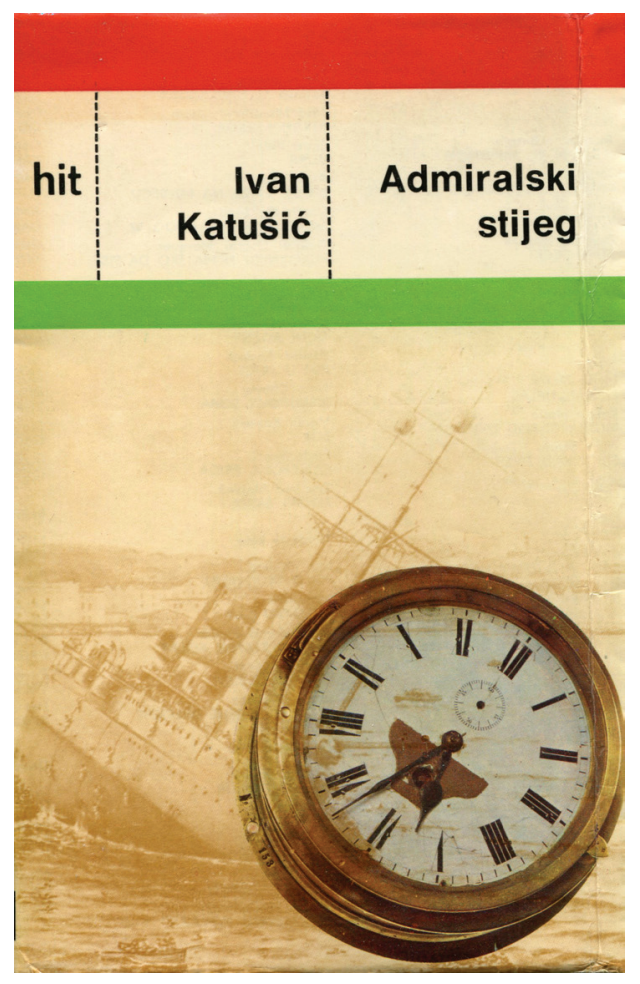

Couverture du roman d'Ivan Katušić, Admiralski stijeg (Zagreb, Znanje, 1987).

62. "Alle Uhren von Viribus Unitis sind „designed in Vienna Forest“ "; «Unser Logo, der Anker, steht für Stabilität und Beständigkeit. In der Form unseres Ankers finden sich die Linien der Buchstaben „Viribus Unitis“ wieder »; «Uhren für echte Männer und angehende Legenden » (viribus-unitis-watches.com, consulté le 14 avril 2019). 